\title{
Tuning of $\mathrm{CeO}_{2}$ buffer layers for coated superconductors through doping
}

\author{
Danny E. P. Vanpoucke ${ }^{\mathrm{a}, \mathrm{b}}$, Stefaan Cottenier ${ }^{\mathrm{c}, \mathrm{d}}$, Veronique Van Speybroeck ${ }^{\mathrm{c}}$, Patrick Bultinck ${ }^{\mathrm{b}}$, Isabel Van Driessche ${ }^{\mathrm{a}}$ \\ ${ }^{a}$ SCRiPTS group, Department of Inorganic and Physical Chemistry, Ghent University, Krijgslaan 281 - S3, BE-9000 Gent, Belgium \\ ${ }^{b}$ Ghent Quantum Chemistry group, Department of Inorganic and Physical Chemistry, Ghent University, Krijgslaan 281 - S3, BE-9000 Gent, Belgium \\ ${ }^{C}$ Center for Molecular Modeling, Ghent University, Technologiepark 903, BE-9052 Zwijnaarde, Belgium \\ ${ }^{d}$ Department of Materials Science and Engineering, Ghent University, Technologiepark 903, BE-9052 Zwijnaarde, Belgium
}

\begin{abstract}
The appearance of microcracks in $\mathrm{CeO}_{2}$ buffer layers, as used in buffer layer architectures for coated superconductors, indicates the presence of stress between this buffer layer and the substrate. This stress can originate from the differences in thermal expansion or differences in lattice parameters between the $\mathrm{CeO}_{2}$ buffer layer and the substrate. In this article, we study, by means of $a b$ initio density functional theory calculations, the influence of group IV doping elements on the lattice parameter and bulk modulus of $\mathrm{CeO}_{2}$. Vegard's law behavior is found for the lattice parameter in systems without oxygen vacancies, and the Shannon crystal radii for the doping elements are retrieved from the lattice expansions. We show that the lattice parameter of the doped $\mathrm{CeO}_{2}$ can be matched to that of the $\mathrm{La}_{2} \mathrm{Zr}_{2} \mathrm{O}_{7}$ coated NiW substrate substrate for dopant concentrations of about $5 \%$, and that bulk modulus matching is either not possible or would require extreme doping concentrations.
\end{abstract}

Keywords: $\mathrm{CeO}_{2}$, doping, lattice parameter, bulk modulus, group IV elements, DFT

\section{Introduction}

Cerium-oxide-based materials have attracted increasing interest over the last two decades. This is mainly due to their remarkable properties with regard to oxidation-reduction catalysis. They are used in a number of industrial applications: threeway catalysts [1], oxygen sensors, solid-oxide fuel cells [2, 3], and many more [4-6]. More recently, cerium oxide $\left(\mathrm{CeO}_{2}\right)$ has been used as thin film buffer layer in $\mathrm{YBa}_{2} \mathrm{Cu}_{3} \mathrm{O}_{7-\delta}$ (YBCO) coated superconductors (CSC) [7-12]. In a YBCO-CSC architecture, a YBCO thin film is grown on a metallic substrate. To prevent the metal atoms of diffusing into the YBCO, one or more buffer layers are required. In addition, these buffer layers also prevent the oxidation of the metallic substrate during YBCO deposition. Due to its structural compatibility with $\mathrm{YBCO}, \mathrm{CeO}_{2}$ is preferred as the top layer in a multilayer architecture. However, the layer thickness of the $\mathrm{CeO}_{2}$ buffer layer is limited by the formation of cracks during deposition [7, 8]. This phenomenon has been linked to internal stress due to lattice mismatch or different thermal expansion coefficients of the substrate and the $\mathrm{CeO}_{2}$ buffer layer [8, 11]. A simple way to reduce the mismatch and stress is through doping [9-11].

In this paper, we study the influence of doping on this mismatch and stress using $a b$ initio atomistic calculations. The energetics and electronic properties of doped systems and the influence of oxygen vacancies is beyond the scope of this work and will be discussed elsewhere.

The stress due to volumetric changes, which are present during the heating and cooling cycles of the production process, are investigated through the bulk moduli (BM) of the doped

Email address: Danny. Vanpoucke@Ugent. be (Danny E. P. Vanpoucke) systems. Because the $\mathrm{CeO}_{2}$ buffer layer is often grown on a $\mathrm{La}_{2} \mathrm{Zr}_{2} \mathrm{O}_{7}$ (LZO) buffer layer in multilayer architectures, a match with the LZO bulk modulus will reduce the inter-layer stress. The change in the $\mathrm{CeO}_{2}$ lattice parameter is derived directly from the calculated atomic structure, and a match with the LZO lattice parameter is searched for to reduce inter-layer stress due to lattice mismatch.

Because $\mathrm{Ce}$ is tetravalent in $\mathrm{CeO}_{2}$, group IV elements are an obvious choice as doping elements. They have the additional advantage that no extra oxygen vacancies need to be introduced for charge compensation. This allows us to retain a clear picture of the direct effects on the lattice parameter and bulk modulus due to the doping elements themselves.

We also look at the effect of aliovalent dopants $(\mathrm{Cu}, \mathrm{Zn}$, and La), assuming oxidized systems without the presence of charge compensating vacancies. This corresponds with experimental systems under (strongly) oxidizing atmosphere. Comparison to results of the group IV elements shows that the valency has relatively little influence.

For such homogeneous systems without oxygen vacancies, it is possible to study the crystal structure from a purely analytic perspective, and we derive Vegard's empirical law analytically for these systems.

\section{Theoretical method}

We perform $a b$ initio density functional theory (DFT) calculations within the projector augmented-wave method as implemented in the Vienna $a b$ initio Package (VASP) program $[13,14]$. The local density approximation (LDA) functional as parameterized by Ceperley and Alder and the generalised gradient approximation (GGA) functional as constructed by Perdew, 

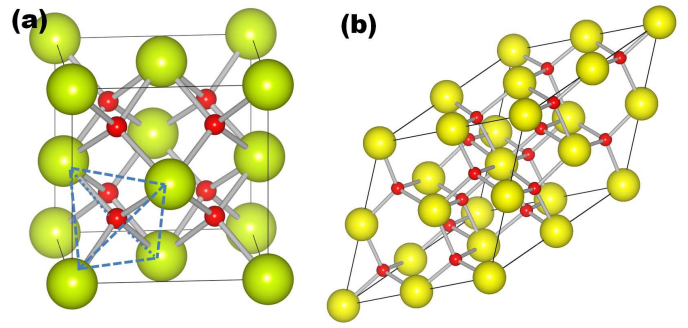

Figure 1: Ball-and-stick model presenting the $\mathrm{CeO}_{2}$ cubic fluorite structure supercells (a) c111 and (b) p222 (cf. text). The big yellow and small red spheres indicate the positions of the $\mathrm{Ce}$ and $\mathrm{O}$ atoms, respectively. The tetrahedral surrounding of a single $\mathrm{O}$ atom is indicated by the tetrahedron for the $\mathrm{c} 111$ supercell.

Burke and Ernzerhof (PBE) are used as exchange-correlation functionals [15-18]. Since our focus goes mainly to the mechanical and structural properties of the system this should be sufficient, and no additional Coulomb correction is required. The plane wave kinetic energy cutoff is set to $500 \mathrm{eV}$.

Symmetric supercells, containing a single dopant per supercell are used to simulate homogeneous distributions of the dopants. The supercells used are the fluorite cubic $1 \times 1 \times 1$ cell with 12 atoms (c111), shown in Fig. 1a, the primitive $2 \times 2 \times 2$ cell with 24 atoms (p222), shown in Fig. 1b, the primitive $3 \times 3 \times 3$ cell with 81 atoms (p333), not shown, and the cubic $2 \times 2 \times 2$ cell with 96 atoms (c222), not shown. Replacing a single Ce atom results in dopant concentrations of 25.0, 12.5,3.7, and 3.1 $\%$, respectively. Monkhorst-Pack special $k$-point grids are used to sample the Brillouin zone [19]. For the two smaller cells we use an $8 \times 8 \times 8 k$-point grid while for the two large supercells a $4 \times 4 \times 4 k$-point grid is used. To optimize the structures, a conjugate gradient method is used. During relaxation both atom positions and cell geometry are allowed to change simultaneously. The convergence criterion is set to the difference in energy between subsequent steps becoming smaller than $1.0 \times 10^{-6} \mathrm{eV}$.

For each dopant the bulk modulus is calculated by fitting $E(V)$ data from fixed volume calculations to the third order isothermal Birch-Murnaghan equation of state [20, 21]. To reduce the computational cost, dopant concentrations of $25 \%$ are used.

Ball-and-stick images of the crystal structures are generated using the VESTA visualization tool [22].

\section{Results and Discussion}

\subsection{Analytic derivation of Vegard's Law}

$\mathrm{CeO}_{2}$ is known to have a cubic fluorite structure (space group $F m \overline{3} m$ ). Figure 1a shows the c111 supercell. Since every O atom is tetrahedrally surrounded by $\mathrm{Ce}$ atoms, the following relation can be derived from the $\mathrm{Ce}-\mathrm{O}$ bond length:

$$
R_{O}+R_{C e}=a_{C e O_{2}} \frac{\sqrt{3}}{4}
$$

with $R_{O}$ and $R_{C e}$ the atomic crystal radii of $\mathrm{O}$ and $\mathrm{Ce}$ respectively, and $a_{\mathrm{CeO}_{2}}$ the $\mathrm{CeO}_{2}$ lattice parameter. For a cubic sys-

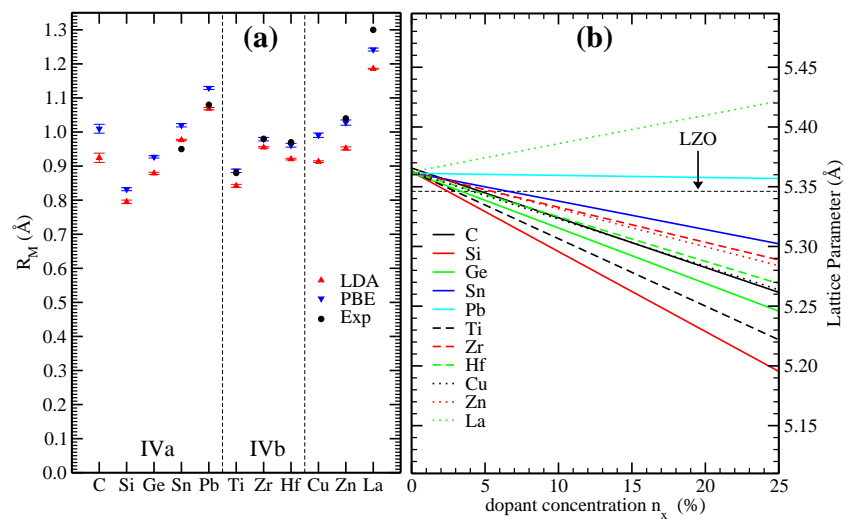

Figure 2: (a) The dopant radii calculated using Eqn. 2 for both the LDA and PBE results. The standard deviation is shown as error bars, and, where available, the experimental Shannon crystal radii $R_{s h}^{8}(c f$. Table 1) are shown as reference. (b) The lattice parameter as function of the dopant concentration, calculated using Eqn. 3. Only the LDA results are shown. The LZO half lattice parameter is shown as reference.

tem, this allows us to calculate the atomic crystal radius $R_{M}$ of a Ce substituent $M$ via:

$$
R_{M}=\left(\frac{\sqrt{3}}{4} a_{M_{x} C e_{1-x} O_{2}}-R_{O}-\left(1-n_{x}\right) R_{C e}\right) / n_{x}
$$

with $a_{M_{x} C e_{1-x} O_{2}}$ the lattice parameter of the doped system and $n_{x}$ the dopant concentration. The lattice parameter of the doped system can now be found by combining Eqns. (1) and (2):

$$
a_{M_{x} C e_{1-x} O_{2}}=a_{C e O_{2}}+\left(\frac{4}{\sqrt{3}}\left(R_{O}+R_{M}\right)-a_{C e O_{2}}\right) n_{x} .
$$

This results in a clear linear relation between the lattice expansion/contraction of $\mathrm{CeO}_{2}$ and the dopant concentration which is known as Vegard's empirical law [23]. These analytical results show Vegard's law behavior should be expected when the dopants are homogeneously distributed in $\mathrm{CeO}_{2}$ in case of tetravalent dopants, but also for non-tetravalent dopants this behavior should be expected under oxidizing conditions.

Although Eqns. (2) and (3) can be derived from one another, the latter is more interesting from the experimental point of view, since lattice parameters and concentrations are readily available while atomic radii are not. Values for the two radii $R_{O}$ and $R_{M}$ can be taken from tabulated values for atomic radii. This, however, can be problematic since there are several different definitions for 'atomic radius' available, giving values which can easily differ $20 \%{ }^{1}$ In light of this problem, Eqn. (2) becomes interesting. It could tell us which definition of atomic radius to use for the doped $\mathrm{CeO}_{2}$ systems. This would allow one to predict the Vegard's law behavior for any doped $\mathrm{CeO}_{2}$ system prior to its synthesis.

\subsection{Group IV elements}

Using Eqn. (2) we have calculated the atomic crystal radius for each of the four concentrations for each group IV element.

\footnotetext{
${ }^{1}$ E.g. for oxygen one finds the calculated atomic radius to be $0.48 \AA$ while the empirical atomic radius is given to be $0.60 \AA[24,25]$.
} 
Table 1: Dopant radii calculated using Eqn. (2), averaged over the four dopant concentrations (avg), and standard deviation (stdev) of this value. This is done for both LDA and PBE calculated geometries. The Shannon crystal radii for the 8-coordinated tetravalent atoms $R_{s h}^{8}$, taken from Ref. [26], are shown in comparison. $a_{0}$ and $b$ are the intercept and slope of the Vegard's law linear fit to the calculated geometries for doped CeO $\mathrm{O}_{2}$ systems. The bulk moduli are calculated for dopant concentrations of $25 \%$. The lattice parameter and bulk modulus (BM) calculated for pure $\mathrm{CeO}_{2}$ and $\mathrm{La}_{2} \mathrm{Zr}_{2} \mathrm{O}_{7}(\mathrm{LZO})$ are given as reference.

\begin{tabular}{|c|c|c|c|c|c|c|c|c|c|c|c|}
\hline & \multicolumn{4}{|c|}{$\mathrm{R}_{M}(\AA)$} & \multirow{3}{*}{$\begin{array}{l}R_{s h}^{8} \\
(\AA)\end{array}$} & \multicolumn{4}{|c|}{ Vegard's Law } & \multicolumn{2}{|c|}{ BM (Mbar) } \\
\hline & \multicolumn{2}{|c|}{ LDA } & \multicolumn{2}{|c|}{ PBE } & & \multicolumn{2}{|c|}{ LDA } & \multicolumn{2}{|c|}{ PBE } & \multirow[t]{2}{*}{ LDA } & \multirow[t]{2}{*}{ PBE } \\
\hline & avg & stdev & avg & stdev & & $a_{0}(\AA)$ & $b$ & $a_{0}(\AA)$ & $b$ & & \\
\hline $\mathrm{CeO}_{2}$ & $1.0819^{a}$ & 0.0001 & $1.1257^{a}$ & 0.0004 & 1.11 & \multirow{2}{*}{\multicolumn{2}{|c|}{$\begin{array}{c}5.3623^{b} \\
10.6923^{c}\end{array}$}} & \multirow{2}{*}{\multicolumn{2}{|c|}{$\begin{array}{c}5.4629^{b} \\
10.8906^{c}\end{array}$}} & 2.017 & 1.715 \\
\hline LZO & & & & & & & & & & 1.774 & 1.542 \\
\hline $\mathrm{C}$ & 0.9243 & 0.0137 & 1.0096 & 0.0130 & - & 5.3656 & -0.4161 & 5.4657 & -0.3077 & 1.528 & 1.235 \\
\hline $\mathrm{Si}$ & 0.7951 & 0.0052 & 0.8321 & 0.0044 & - & 5.3626 & -0.6688 & 5.4643 & -0.6936 & 2.057 & 1.738 \\
\hline $\mathrm{Ge}$ & 0.8786 & 0.0031 & 0.9270 & 0.0034 & - & 5.3618 & -0.4640 & 5.4631 & -0.4578 & 1.909 & 1.573 \\
\hline $\mathrm{Sn}$ & 0.9764 & 0.0020 & 1.0199 & 0.0049 & 0.95 & 5.3618 & -0.2383 & 5.4629 & -0.2400 & 2.004 & 1.692 \\
\hline $\mathrm{Pb}$ & 1.0686 & 0.0034 & 1.1293 & 0.0042 & 1.08 & 5.3612 & -0.0174 & 5.4633 & 0.0050 & 1.845 & 1.516 \\
\hline $\mathrm{Ti}$ & 0.8421 & 0.0043 & 0.8862 & 0.0050 & 0.88 & 5.3629 & -0.5640 & 5.4644 & -0.5706 & 2.145 & 1.825 \\
\hline $\mathrm{Zr}$ & 0.9548 & 0.0019 & 0.9791 & 0.0052 & 0.98 & 5.3622 & -0.2938 & 5.4634 & -0.3409 & 2.153 & 1.878 \\
\hline Hf & 0.9205 & 0.0023 & 0.9612 & 0.0056 & 0.97 & 5.3622 & -0.3733 & 5.4635 & -0.3849 & 2.194 & 1.881 \\
\hline $\mathrm{Cu}$ & 0.9131 & 0.0024 & 0.9907 & 0.0064 & $0.91^{e} /-^{h}$ & 5.3624 & -0.3947 & 5.4627 & -0.3065 & 1.704 & 1.374 \\
\hline & & & & & & & & & & $1.867^{d}$ & $1.553^{d}$ \\
\hline $\mathrm{Zn}$ & 0.9518 & 0.0046 & 1.0277 & 0.0077 & $0.88^{f} / 1.04^{i}$ & 5.3632 & -0.3173 & 5.4640 & -0.2389 & 1.712 & 1.410 \\
\hline $\mathrm{La}$ & 1.1858 & 0.0011 & 1.2421 & 0.0043 & $1.17^{g} / 1.30^{j}$ & 5.3623 & 0.2374 & 5.4637 & 0.2597 & 1.835 & 1.556 \\
\hline
\end{tabular}

${ }^{a}$ The Ce radius is calculated using Eqn. (1), where the 4-coordinated Shannon crystal radius for oxygen is taken as $1.24 \AA$ [26].

${ }^{b},{ }^{c}$ The actual lattice parameter as calculated from the pure, relaxed geometries of $\mathrm{CeO}_{2}$ and LZO. Note that the LZO lattice parameter is double the $\mathrm{CeO}_{2}$ lattice parameter.

${ }^{d}$ The bulk modulus for $\mathrm{Cu}$ dopant concentration of $12.5 \%$.

${ }^{e},{ }^{f},{ }^{g}$ Shannon crystal radii for 6-coordinated $\mathrm{Cu}^{1+}, \mathrm{Zn}^{2+}$, and $\mathrm{La}^{3+}$.

${ }^{h},{ }^{i},{ }^{j}$ Shannon crystal radii for 8 -coordinated $\mathrm{Cu}^{1+}, \mathrm{Zn}^{2+}$, and $\mathrm{La}^{3+}$.

The average values and their standard deviations are shown in Table 1 and Fig. 2a. In these calculations we have used the Shannon crystal radius for four-coordinate $\mathrm{O}^{2-} ; R_{O}=1.24$ $\AA$. The radius of eight-coordinate $\mathrm{Ce}^{4+}$ is calculated from the non-doped $\mathrm{CeO}_{2}$ system using Eqn. (1) and shows very good agreement with the Shannon crystal radius for eight-coordinate $\mathrm{Ce}^{4+}[26,27]$. For the group IV elements, the LDA and PBE calculations show the same relative trends, with the PBE values always slightly larger than the LDA ones, as one would expect. Due to the underbinding nature of GGA functionals such as PBE and the overbinding nature of LDA, we expect the LDA and PBE values to be a slight under- and overestimation of the actual crystal radius, respectively. Comparison of the calculated dopant radii to the Shannon crystal radii for eight-coordinated tetravalent atoms $R_{s h}^{8}$ shows a very good correlation. This indicates that the Shannon crystal radius is an excellent parametrization to predict the lattice expansion of doped $\mathrm{CeO}_{2}$.

Based on lattice parameters obtained from the $a b$ initio calculations for the different dopant concentrations, a fitting of Vegard's law is done for each of the group IV elements. Table 1 shows the intercept $a_{0}$ and the slope $b$ of this linear fitting. For the LDA results, the fitted curves of the lattice parameter as function of the dopant concentration $n_{x}$ are shown in Fig. 2b. With the exception of $\mathrm{Pb}$, all systems show a nearly perfect fit, with correlation coefficient $\left(R^{2}\right)$ values better than 0.99 for both LDA and PBE calculations. The poor fit of $\mathrm{Pb}$ is related to the negligible expansion of the lattice. As a result, small deviations can transform an expansion into a contraction going from one concentration to the next. A zero expansion can thus be assumed for $\mathrm{Pb}$ doping. The high quality of the other linear fits, with intercepts that are within $0.01 \AA$ of the $\mathrm{CeO}_{2}$ lattice parameter, shows that the analytically obtained Vegard's law is a good model for the lattice expansion in these doped systems.

Both LDA and PBE results show the same qualitative behavior, and the optimum substitution concentrations, for lattice parameter matching with LZO, are $\sim 5 \%$ for all group IV elements, with, due to its negligible contraction, the exception of $\mathrm{Pb}$ substitution.

Table 1 also shows the bulk modulus, obtained for substituent concentrations of $25 \%$. If we assume the bulk modulus to behave linearly with regard to the dopant concentration ( $c f$. next section), then it is possible to estimate the optimum dopant concentration which would result in a perfect matching of the bulk modulus of the doped $\mathrm{CeO}_{2}$ and LZO. For the elements of group IVb, table 1 shows a bulk modulus which is larger than 
that of $\mathrm{CeO}_{2}$. This makes bulk modulusmatching with LZO, which has a smaller bulk modulus, impossible. In addition, the bulk modulus seems to increase very slightly with increasing atomic number. The group IVa elements show a more complex behavior. With the exception of $\mathrm{Si}$, all group IVa elements lower the bulk modulus of $\mathrm{CeO}_{2}$. However, the effect is generally too small to allow for a bulk modulus matching at reasonable dopant concentrations. In case of the IVa elements, the bulk modulus is lowered with the introduction of every newly filled shell ( $d$ for $\mathrm{Ge}$ and $\mathrm{Sn}$, and $f$ for $\mathrm{Pb}$ ), while it increases with increasing atomic radii. Combining the results of the groups IVa and IVb shows that not only the valency electrons but also electrons in filled shells near the Fermi level play a crucial role for the bulk modulus.

\subsection{Aliovalent dopants without vacancies}

We have also investigated the effect of doping with aliovalent elements, but this without the introduction of charge compensating vacancies. This makes comparison between aliovalent and group IV elements more straightforward. The three aliovalent elements we have investigated are: $\mathrm{Cu}, \mathrm{Zn}$, and La. (The effect of the addition of charge compensating vacancies is a topic on its own, and will therefore be discussed elsewhere.)

If we look at the calculated atomic radii in Table 1, we see that unlike with the group IV elements, the values are smaller than the Shannon crystal radii for 8-coordinated atoms, but larger than the values for 6 -coordinated atoms. ${ }^{2}$ This would indicate that the lower valence results in a lower coordination, regardless of the geometric and chemical surrounding. This in turn should give rise to charge redistribution near the defect.

Just as for the group IV elements, the obtained lattice parameters for different concentrations can be fitted nicely against a linear Vegard law, with $R^{2}$ values better than 0.99 . This means that under oxidizing atmosphere, i.e. with little or no oxygen vacancies in the system, also aliovalent dopants should show a linear concentration dependence of the lattice parameter. As for the group IV elements lattice parameter matching with LZO is found to be $\sim 5 \%$ for $\mathrm{Cu}$ and $\mathrm{Zn}$. For La doping, which shows a lattice expansion, no lattice parameter matching with LZO is possible.

In case of $\mathrm{Cu}$, we calculated the bulk modulus for dopant concentrations of 25.0 and $12.5 \%$, shown in Table 1. Taking the $\mathrm{CeO}_{2}$ bulk modulus as the case of $0.0 \%$ doping, a nearly linear trend is observed. Although all three aliovalent elements show a decrease of the bulk modulus which looks slightly better than is the case for the group IV elements, very high dopant concentrations would still be needed to have bulk modulus matching with LZO.

Combined with the results for the group IV elements, it is clear that the lattice parameter is only influenced by the (Shannon) crystal radius of the doping elements, while the bulk modulus is also strongly influenced by the electronic structure (compare $\mathrm{Zn} / \mathrm{Zr}$ and $\mathrm{Hf} / \mathrm{Cu}$ ).

\footnotetext{
${ }^{2}$ Extrapolating the tabulated Shannon crystal radius values for $\mathrm{Cu}^{1+}$ to the coordination number 8 would give a value in the range of $1.05-1.11 \AA$.
}

\section{Conclusion}

The influence of group IV and aliovalent dopants on the lattice parameter and bulk modulus of $\mathrm{CeO}_{2}$ are investigated using DFT calculations. A Vegard's law relation is analytically derived for doped $\mathrm{CeO}_{2}$ without oxygen vacancies. lattice parameters obtained from DFT calculations for different dopant concentrations show that for both group IV and aliovalently doped systems without oxygen vacancies the lattice expansion is described by a linear relation. This Vegard's law can be predicted from the Shannon crystal radius of the dopant element. Optimum doping concentrations for lattice parameter matching with LZO is about $5 \%$ for the different dopants studied, the exceptions being $\mathrm{Pb}$ and $\mathrm{La}$. The former shows no appreciable expansion of contraction of the $\mathrm{CeO}_{2}$ lattice parameter, while the latter results in a lattice expansion. It is shown that group IVb dopants result in a slight increase in the bulk modulus, while group IVa, except $\mathrm{Si}$, and the aliovalent dopants show a decrease in the bulk modulus of $\mathrm{CeO}_{2}$. The decrease, however, is insufficient to obtain bulk modulus matching at concentrations similar to those needed for lattice parameter matching with LZO.

\section{Acknowledgement}

The research was financially supported by FWO-Vlaanderen, project $\mathrm{n}^{\circ}$ 3G080209, EMRS Symposium A organization and FWO (grant K1B9711N). We acknowledge the Research Board of the Ghent University.S. C. acknowledges financial support from OCAS NV by an OCAS-endowed industrial chair at Ghent University. This work was carried out using the Stevin Supercomputer Infrastructure at Ghent University.

\section{References}

[1] F. Deganello, A. Martorana, J. Solid State Chem. 163 (2002) 527-533.

[2] H. L. Tuller, A. S. Nowick, J. Electrochem. Soc. 122 (1975) 255-259.

[3] T. Miki, T. Ogawa, M. Haneda, N. Kakuta, A. Ueno, S. Tateishi, S. Matsuura, M. Sato, J. Phys. Chem. 94 (1990) 6464-6467.

[4] Lj. Kundakovic, M. Flytzani-Stephanopoulos, Journal of Catalysis 179 (1998) $203-221$.

[5] Y. She, Q. Zheng, L. Li, Y. Zhan, C. Chen, Y. Zheng, X. Lin, Int. J. Hydrogen Energy 34 (2009) 8929-8936.

[6] M. Manzoli, G. Avgouropoulos, T. Tabakova, J. Papavasiliou, T. Ioannides, F. Boccuzzi, Catal. Today 138 (2008) $239-243$.

[7] M. Paranthaman, A. Goyal, F. List, E. Specht, D. Lee, P. Martin, Q. He, D. Christen, D. Norton, J. Budai, D. Kroeger, Physica C 275 (1997) 266 -272 .

[8] S. Oh, J. Yoo, K. Lee, J. Kim, D. Youm, Physica C 308 (1998) 91 - 98.

[9] Y. Takahashi, Y. Aoki, T. Hasegawa, T. Maeda, T. Honjo, Y. Yamada, Y. Shiohara, Physica C 412-414, Part 2 (2004) 905-909.

[10] K. Knoth, B. Schlobach, R. Hühne, L. Schultz, B. Holzapfel, Physica C 426-431, Part 2 (2005) 979-984.

[11] N. Van de Velde, D. Van de Vyver, O. Brunkahl, S. Hoste, E. Bruneel, I. Van Driessche, Eur. J. Inor. Chem. (2010) 233-241.

[12] G. Penneman, I. Van Driessche, E. Bruneel, S. Hoste, in: Mandal, H and Ovecoglu, L (Ed.), Euro Ceramics VIII, Pts 1-3, volume 264-268 of Key Engineering Materials, Turkish Ceram Soc; European Ceram Soc, pp. 501-504. 8th Conference of the European-Ceramic-Society, Istanbul, Turkey, jun 29-jul 03, 2003.

[13] P. E. Blöchl, Projector augmented-wave method, Phys. Rev. B 50 (1994) $17953-17979$. 
[14] G. Kresse, D. Joubert, Phys. Rev. B 59 (1999) 1758-1775.

[15] D. M. Ceperley, B. J. Alder, Phys. Rev. Lett. 45 (1980) 566-569.

[16] J. P. Perdew, K. Burke, M. Ernzerhof, Phys. Rev. Lett. 77 (1996) 38653868.

[17] G. Kresse, J. Hafner, Phys. Rev. B 47 (1993) 558-561.

[18] G. Kresse, J. Furthmüller, Phys. Rev. B 54 (1996) 11169-11186.

[19] H. J. Monkhorst, J. D. Pack, Phys. Rev. B 13 (1976) 5188-5192.

[20] F. D. Murnaghan, Proc. Natl. Acad. Sci. USA 30 (1944) 244-247.

[21] F. Birch, Phys. Rev. 71 (1947) 809-824.

[22] K. Momma, F. Izumi, J. Appl. Cryst. 41 (2008) 653-658

[23] A. R. Denton, N. W. Ashcroft, Phys. Rev. A 43 (1991) 3161-3164.

[24] E. Clementi, D. L. Raimondi, J. Chem. Phys. 38 (1963) 2686.

[25] J. Slater, J. Chem. Phys. 41 (1964) 3199.

[26] J. D. Van Horn, Electronic Table of Shannon Ionic Radii, 2001. http://v.web.umkc.edu/vanhornj/shannonradii.htm, Downloaded 08/13/2010.

[27] R. D. Shannon, Acta Cryst. A32 (1976) 751-767. 